\title{
Differential Aging Trajectories of Modulation of Activation to Cognitive Challenge in APOE $\varepsilon 4$ Groups: Reduced Modulation Predicts Poorer Cognitive Performance
}

\author{
(DChris M. Foster, ${ }^{-K}$ Kristen M. Kennedy, and $\odot$ Karen M. Rodrigue \\ Center for Vital Longevity, School of Behavioral and Brain Sciences, The University of Texas at Dallas, Dallas, Texas 75235
}

The present study was designed to investigate the effect of a genetic risk factor for Alzheimer's disease (AD), ApolipoproteinE $\varepsilon 4$ (APOE 84 ), on the ability of the brain to modulate activation in response to cognitive challenge in a lifespan sample of healthy human adults. A community-based sample of 181 cognitively intact, healthy adults were recruited from the Dallas-Fort Worth metroplex. Thirty-one APOE $\varepsilon 4+$ individuals ( $48 \%$ women), derived from the parent sample, were matched based on sex, age, and years of education to 31 individuals who were $\mathrm{APOE} \varepsilon 4$-negative (APOE $\varepsilon 4-)$. Ages ranged from 20 to 86 years of age. Blood oxygen level-dependent functional magnetic resonance imaging was collected during the performance of a visuospatial distance judgment task with three parametric levels of difficulty. Multiple regression was used in a whole-brain analysis with age, APOE group, and their interaction predicting functional brain modulation in response to difficulty. Results revealed an interaction between age and APOE in a large cluster localized primarily to the bilateral precuneus. APOE $\varepsilon 4-$ individuals exhibited age-invariant modulation in response to task difficulty, whereas APOE $\varepsilon 4+$ individuals showed age-related reduction of modulation in response to increasing task difficulty compared with $\varepsilon 4-$ individuals. Decreased modulation in response to cognitive challenge was associated with reduced task accuracy as well as poorer name-face associative memory performance. Findings suggest that APOE $\varepsilon 4$ is associated with a reduction in the ability of the brain to dynamically modulate in response to cognitive challenge. Coupled with a significant genetic risk factor for $\mathrm{AD}$, changes in modulation may provide additional information toward identifying individuals potentially at risk for cognitive decline associated with preclinical AD.

Key words: aging; APOE; cognition; fMRI

Significance Statement

Understanding how risk factors for Alzheimer's disease (AD) affect brain function and cognition in healthy adult samples may help to identify the biomarkers needed to detect nonsymptomatic, preclinical phases of the disease. Findings from the current study show that ApolipoproteinE $\varepsilon 4$-positive $(\mathrm{APOE} \varepsilon 4+$ ) individuals exhibit an altered lifespan trajectory in the ability of the brain to dynamically modulate function to cognitive challenge compared with APOE $\varepsilon 4-$ individuals. This effect manifests in otherwise healthy individuals who are at increased risk for $\mathrm{AD}$ in the precuneus, a salient region for early $\mathrm{AD}$ changes. Notably, these functional alterations are detrimental to performance, and thus, the combination of a genetic risk factor and altered modulation may provide important information for identifying individuals who are at increased risk for AD.

\section{Introduction}

The ApolipoproteinE $\varepsilon 4$ (APOE $\varepsilon 4$ ) allele is the strongest known genetic risk factor for sporadic Alzheimer's disease (AD; Corder

\footnotetext{
Received Dec. 20, 2016; revised June 1, 2017; accepted June 8, 2017.

Author contributions: K.M.K. and K.M.R. designed research; K.M.K. and K.M.R. performed research; C.M.F., K.M.K., and K.M.R. analyzed data; C.M.F., K.M.K., and K.M.R. wrote the paper.

This research was supported in part by National Institute on Aging Grant AG-036848 to K.M.R, and Grant AG-036818 to K.M.K.

The authors declare no competing financial interests.

Correspondence should be addressed to Dr. Karen M. Rodrigue, Center for Vital Longevity, 1600 Viceroy Drive,

Suite 800, Dallas, TX 75235. E-mail: krodrigue@utdallas.edu.
}

et al., 1993). Structural changes in APOE protein impact its ability to bind with lipids and $\beta$-amyloid and to reduce its functional ability to maintain neuronal health (Frieden and Garai, 2012; Liu et al., 2013). Indeed, $\varepsilon 4$-positive $(\varepsilon 4+)$ individuals are more likely to accumulate extracellular $\beta$-amyloid $(\mathrm{A} \beta)$ protein, as the $\mathrm{APOE} \varepsilon 4$ isoform reduces $\mathrm{A} \beta$ clearance and increases $\mathrm{A} \beta$ aggregation (Verghese et al., 2011). Without adequate clearance, $\beta$-amyloid can form oligomers and plaque, which are thought to be neuro- 
Table 1. Participant demographics

\begin{tabular}{llllllllll}
\hline APOE group & $N$ (\% women) & Age & Education & MMSE & CES-D & CFIT & Vocab & MfN & VPA \\
\hline$\epsilon 4^{-}$ & $31(48)$ & $53.68(19.92)$ & $15.48(2.73)$ & $28.77(0.85)$ & $3.55(3.09)$ & $6.65(1.48)$ & $26.45(4.40)$ & $22.45(9.20)$ & $10.55(3.39)$ \\
$\epsilon 4^{+}$ & $31(48)$ & $53.94(19.96)$ & $14.71(2.81)$ & $29.03(0.75)$ & $4.13(3.40)$ & $6.43(1.43)$ & $25.29(5.20)$ & $22.68(8.86)$ & $11.36(2.50)$ \\
Total & $62(48)$ & $53.81(19.78)$ & $15.10(2.77)$ & $28.90(0.80)$ & $3.84(3.24)$ & $6.54(1.45)$ & $25.87(4.81)$ & $22.57(8.96)$ & $10.95(2.98)$ \\
\hline
\end{tabular}

Values are the mean (SD), unless otherwise indicated. Vocab, Educational Testing Service Vocabulary subtest; MfN, Memory for Names Delayed subtest; VPA, Verbal Paired Associates Cued Recall subtest. There were no significant APOE group differences ( $p$ values $>0.206$ ) or APOE by age interactions ( $p$ values $>0.133$ ) on any demographic or screening measure. Negative age effects were present for CES-D $(p=0.037), \operatorname{MfN}(p=0.001)$, CFIT ( $p=0.002)$, and VPA $(p<0.001)$.

toxic to neurons (Liu et al., 2013; Scheltens et al., 2016). Thus, $\mathrm{APOE} \varepsilon 4$ carriers are likely to show reduced efficiency in neural function, and fMRI evidence suggests that differences in taskrelated brain function are apparent in even younger adults (Filippini et al., 2009, 2011; Dennis et al., 2010; Matura et al., 2014; Suri et al., 2015), far preceding the development of AD.

However, healthy aging is also associated with changes in functional activation to cognitive tasks. One key observation in aging studies is a reduction in the ability of the brain to respond to cognitive challenges (Reuter-Lorenz and Cappell, 2008). Specifically, older adults show decreased capacity to modulate brain activation to more difficult task levels (Schneider-Garces et al., 2010; Kennedy et al., 2015a; Rieck et al., 2017), reflecting a reduction in the ability of the aging brain to dynamically respond to challenge, potentially representing the neural equivalent of a processing resource ceiling (Craik and Byrd, 1982). Interestingly, within older adults, those who better maintain the ability to modulate the blood oxygenation level-dependent (BOLD) response to difficulty exhibit higher fluid reasoning ability (Rieck et al., 2017), suggesting that altered modulation is also associated with poorer cognition. However, there may be modifying factors underlying age effects that either ameliorate or exacerbate the decreased modulation in response to cognitive challenge. Given the effect of APOE on neuronal health, $\varepsilon 4$ carriers may be at risk for the depletion of neuronal resources and therefore may show decreased ability to flexibly modulate brain activity to a greater extent or at an earlier age than noncarriers.

Existing studies examining the effect of APOE on functional brain activation to cognitive challenge used a working memory $n$-back task and generally found that older $\varepsilon 4$ carriers exhibited less modulation compared with non- $\varepsilon 4$ carriers on the hardest level of the task (Wishart et al., 2006; Chen et al., 2013; Yan et al., 2015). Of the two studies of younger adults, one found greater activation in $\varepsilon 4$ carriers (Shine et al., 2015) and one found no activation differences between carrier groups (Mondadori et al., 2007), leaving open the question as to how APOE affects modulation in response to cognitive challenge across the lifespan. Further, it is unclear whether activation differences were beneficial or detrimental to cognitive performance as no associations with behavior were reported.

Thus, the present study was designed to investigate the effects of APOE, a salient genetic risk factor for AD, on the ability of the brain to modulate activation in response to cognitive challenge in a sample of healthy adults. We sought to extend the prior literature in several ways. First, our lifespan approach allowed for more complete understanding of when APOE $\varepsilon 4$ exerts adverse effects on BOLD modulation. Second, we used a visuospatial distance judgment task, that varied in difficulty, to isolate age-related changes in response to cognitive challenge, as opposed to changes in working memory per se. Finally, we sought to investigate whether BOLD modulation was related to behavior, both on the in-scanner task and, given the strong connection between APOE $\varepsilon 4$ and memory changes in older adults (for review, see Liu et al., 2013; Sperling et al., 2014), on associative memory performance outside of the scanner. We hypothesized that APOE $\varepsilon 4$ carriers would show greater decline in modulation with aging compared to a demographically matched group of $\varepsilon 4$-negative $(\varepsilon 4-)$ adults. We also predicted that decreased modulation would be detrimental to these indices of cognitive performance.

\section{Materials and Methods}

\section{Participants}

From a larger parent study of 181 individuals, participants in the current study were selected for analysis in a case-control matched design. Of the parent sample, 31 individuals were homozygous or heterozygous for APOE $\varepsilon 4$. We then matched these 31 APOE $\varepsilon 4+$ individuals $(\varepsilon 4 / \varepsilon 4, n=$ $1 ; \varepsilon 2 / \varepsilon 4, n=3 ; \varepsilon 3 / \varepsilon 4, n=27$ ) with 31 individuals who were APOE $\varepsilon 4-$ $(\varepsilon 2 / \varepsilon 3, n=8 ; \varepsilon 3 / \varepsilon 3, n=23)$ based on sex, age, and years of education. We opted to retain all $\varepsilon 4$ heterozygotes, including $\varepsilon 2 / \varepsilon 4$ individuals, as any individual with an $\varepsilon 4$ allele is at greater risk of $\mathrm{AD}$ than individuals without $\varepsilon 4$ alleles (Liu et al., 2013). Results are unchanged when these individuals are removed from analyses. Therefore, the current study included 62 healthy adults (mean age, $53.81 \pm 19.78$ years; age range, 20-86 years; Table 1). Participants were recruited from the Dallas-Fort Worth metroplex and were screened to ensure they were right handed, fluent English speakers, and had normal or corrected-to-normal vision. If required, MRI-compatible glasses were used on the day of scanning. Participants were screened to be free from a history of neurological or psychiatric conditions, head trauma, drug or alcohol problems, and significant cardiovascular disease, and to be cognitively intact [Mini-Mental State Examination (MMSE) score, $\geq 26$; Folstein et al., 1975] and not depressed (Center for Epidemiological Studies-Depression (CES-D) scale score, <16; Radloff, 1977). Seventeen participants self-reported a diagnosis of hypertension. Participants were required to score at or above chance on the control condition of the in-scanner task. As a measure of fluid intelligence, participants completed the Culture Fair Intelligence Test (CFIT; Cattell and Cattell, 1963), and as a measure of crystalized intelligence participants completed the Vocabulary Test II-V2 (Educational Testing Service, 1962). There were no significant differences between APOE $\varepsilon 4-$ and APOE $\varepsilon 4+$ individuals in any demographic or screening variables, including age, education, and scores on the MMSE, CES-D, CFIT, or Vocabulary Test II-V2 (all $p$ values $>0.206$; Table 1 ). Informed consent was obtained in accordance with the protocol approved by the University of Texas at Dallas and the University of Texas Southwestern Medical Center.

\section{Genetic sequencing}

For a detailed description of the genetic sequencing protocol, see the study by Kennedy et al. (2015b). In brief, saliva samples were collected using Oragene kits during a neuropsychological testing visit before MRI scanning. DNA extraction and genotyping were conducted at the University of Texas Southwestern Medical Center Microarray Core Facility. APOE information (rs429358 and rs7412) was acquired using TaqMan SNP Genotyping assays (Applied Biosystems). DNA genotyping reactions were performed using the $0.5 \times$ protocol with the Applied Biosystems TaqMan Genotyping Master Mix.

\section{Neuropsychological testing}

On a separate visit before scanning (mean, $50 \mathrm{~d} ; \mathrm{SD}=37 \mathrm{~d}$ ), participants completed a battery of neuropsychological assessments, which included two associative memory tests: the Memory for Names subtest from the Woodcock-Johnson III Psychoeducational Battery (Woodcock et al., 2007) and the Verbal Paired Associates test (Wechsler, 2009). The Mem- 
Table 2. Visuospatial distance judgment task behavioral performance

\begin{tabular}{|c|c|c|c|c|c|c|}
\hline \multirow[b]{2}{*}{ APOE allele } & \multicolumn{3}{|c|}{ Mean accuracy } & \multicolumn{3}{|c|}{ Median response time (s) } \\
\hline & Easy & Medium & Hard & Easy & Medium & Hard \\
\hline$\epsilon 4^{-}$ & $94.09(9.20)$ & $87.25(14.00)$ & $72.81(18.21)$ & $0.85(0.16)$ & $0.89(0.14)$ & $1.02(0.23)$ \\
\hline$\epsilon 4^{+}$ & $95.54(8.04)$ & $92.08(11.58)$ & $66.50(19.91)$ & $0.79(0.13)$ & $0.85(0.16)$ & $1.03(0.22)$ \\
\hline Total & $94.82(8.60)$ & $89.67(12.98)$ & $69.66(19.18)$ & $0.82(0.15)$ & $0.87(0.15)$ & $1.02(0.22)$ \\
\hline
\end{tabular}

Values are mean (SD).

ory for Names test is a measure of associative memory where participants learn pairings of novel space creature drawings to their nonsense names (i.e., Plik) and are tested immediately after learning and again after a 30 min delay. Delayed memory scores index the total number of correctly identified associations. The Verbal Paired Associates test requires participants to learn word pairs over repeated trials with both immediate and delayed tests. The Memory for Names Delay and Verbal Paired Associates tests were specifically chosen as a neuropsychological measure of interest due to the fact that associative memory has been established as a key marker for both normal aging and unhealthy aging (NavehBenjamin, 2000; Naveh-Benjamin et al., 2003; Sperling et al., 2014). Further, the Memory for Names test is also a name-face learning task, a cognitive function that is often reported as the most common memory complaint among older adults (Naveh-Benjamin et al., 2004).

\section{MRI Acquisition}

All participants were scanned using a single Philips Achieva $3 \mathrm{~T}$ wholebody scanner equipped with a 32 -channel head coil in a $75 \mathrm{~min}$ session. High-resolution anatomical images were collected with a T1-weighted MP-RAGE sequence with the following parameters: 160 sagittal slices; $1 \times 1 \times 1 \mathrm{~mm}^{3}$ voxels; $256 \times 204 \times 160$ matrix; FOV $=256 \mathrm{~mm}$; TE $=$ $3.8 \mathrm{~ms}$; TR $=8.3 \mathrm{~ms}$; flip angle $=12^{\circ}$. BOLD fMRI data were acquired using a T2*-weighted echoplanar imaging sequence in 29 interleaved axial slices parallel to the anterior commissure-posterior commissure line $\left(64 \times 64 \times 29\right.$ matrix; $3.4 \times 3.4 \times 5 \mathrm{~mm}^{3}$; FOV $=220 \mathrm{~mm}$; TE $=$ $30 \mathrm{~ms}$; TR $=1500 \mathrm{~ms})$.

\section{Experimental design and statistical analysis}

fMRI distance judgment task. For a detailed description of the distance judgment task see Rieck et al. (2017). In brief, after pretraining on the task outside the scanner, participants performed a coordinate judgment task using three parametric levels of difficulty (easy, medium, hard) as well as a categorical judgment task used as a control condition (modeled after the study by Baciu et al., 1999; and adapted from the study by Park et al., 2010). To minimize the time between practice and performance of the task, participants completed the distance judgment task immediately upon entering the scanner. In a blocked design, participants were shown, in the categorical condition, a dot appearing to the right or left of a horizontal bar and indicated on each trial with a button press on which side of the bar the dot appeared. In the coordinate judgment condition, participants were first presented with a vertical reference line and then presented with the horizontal bar with the dot some parametric distance above or below the horizontal bar. The participant indicated on each trial with a button press whether the dot was "Nearer" to or "Farther" away from the horizontal bar than the length of the vertical reference line. Three 5 min runs were completed by each participant. Each run contained 20 blocks and contained all four conditions of the task presented in a pseudorandom order. Each of the 20 blocks contained five trials and lasted $15 \mathrm{~s}$. Spaced across the three runs, there was a total of 15 easy, medium, and hard coordinate task blocks along with seven categorical control task blocks.

fMRI processing. The time series data for individual participants were preprocessed with Statistical Parametric Mapping 8 (SPM8; Wellcome Department of Cognitive Neurology, London, UK) according to a standard pipeline of procedures. In order, images were corrected for differences in slice time acquisition, individual volumes were corrected for within-run participant movement, and finally, images were normalized to a common MNI space and smoothed with an isotropic $8 \mathrm{~mm}^{3}$ fullwidth at half-maximum Gaussian kernel. ArtRepair (Mazaika et al., 2009) was also used to identify outlier volumes for each participant. For each participant, runs that had $>15 \%$ outlier volumes ( $\sim 30$ volumes) with $>3 \%$ deviation from the mean in global intensity spikes or $>2 \mathrm{~mm}$ of motion displacement were flagged. Two participants had one run of data (of three runs) flagged for excessive outlier volumes, and the noisy run was subsequently removed from further analysis.

$f M R I$ data analysis. At the individual subject level, the BOLD response to each condition (control, easy, medium, hard) was modeled as a block convolved with a canonical hemodynamic response function; six directions of motion estimates for each volume generated from ArtRepair were also included as nuisance covariates. For each participant, a parametric linear difficulty contrast of hard versus medium versus easy was computed, and this contrast was used as a measure of the modulation of activation (hard greater than medium greater than easy) or modulation of deactivation (easy greater than medium greater than hard) in response to cognitive challenge.

To test for the effects of age, APOE genotype, and their interaction on BOLD modulation in response to task difficulty, a linear regression was conducted that included an intercept term, a continuous mean-centered age factor, a dummy-coded grouping factor such that the $\varepsilon 4-$ group was the reference group, an age by APOE genotype interaction term, and mean centered average task accuracy. In this model, the intercept term represents the simple effect of task difficulty for the $\varepsilon 4-$ group, and the age factor represents the simple effect of age for the $\varepsilon 4-$ group. The dummy-coded grouping factor represents $\varepsilon 4+$ differences in response to task difficulty, and the age by APOE interaction represents $\varepsilon 4+$ differences in slopes across age. All results were thresholded using an FWE cluster correction of $p<0.05$ and an uncorrected height threshold of $p<0.01$. To ensure that type I error rates were correctly controlled at the cluster level (Eklund et al., 2016), cluster corrections were calculated using the Statistical nonParametric Mapping toolbox (SnPM13; http:// warwick.ac.uk/snpm). Five thousand permutations were conducted to derive the corrected clusterwise threshold. No individual parameter estimate was found to be greater than 3 SDs away from the mean. The fMRI analysis was performed with and without the four non- $\varepsilon 3 / \varepsilon 4$ individuals and their matched $\varepsilon 4-$ counterparts, and the pattern of results was unchanged. Thus, the reported results include all participants.

\section{Results}

\section{Task performance}

Mean accuracy and median response time were calculated for each participant for easy, medium, and hard conditions. Two general linear models were run with accuracy or response time for each condition as a within-subject repeated measure. Betweensubjects measures included age as a continuous variable and APOE as a categorical variable. The three-way interaction among task difficulty, age, and APOE, as well as all subordinate two-way interactions were entered into the model. For accuracy, there was a significant effect of task difficulty $\left(F_{(2,116)}=14.01, p<0.001\right.$, $\left.\eta_{p}^{2}=0.195\right)$, such that accuracy decreased as task difficulty increased. No other effects reached significance ( $p$ values $>0.144$; Table 2). The response time analysis produced similar results: there was a significant main effect of task difficulty $\left(F_{(2,116)}=\right.$ 14.19, $\left.p<0.001, \eta_{p}^{2}=0.197\right)$ as well as a marginally significant effect of age $\left(F_{(1,58)}=3.63, p=0.062, \eta_{p}^{2}=0.059\right)$, indicating that response times significantly slowed as task difficulty increased and that there was a trend for a decrease in response time 


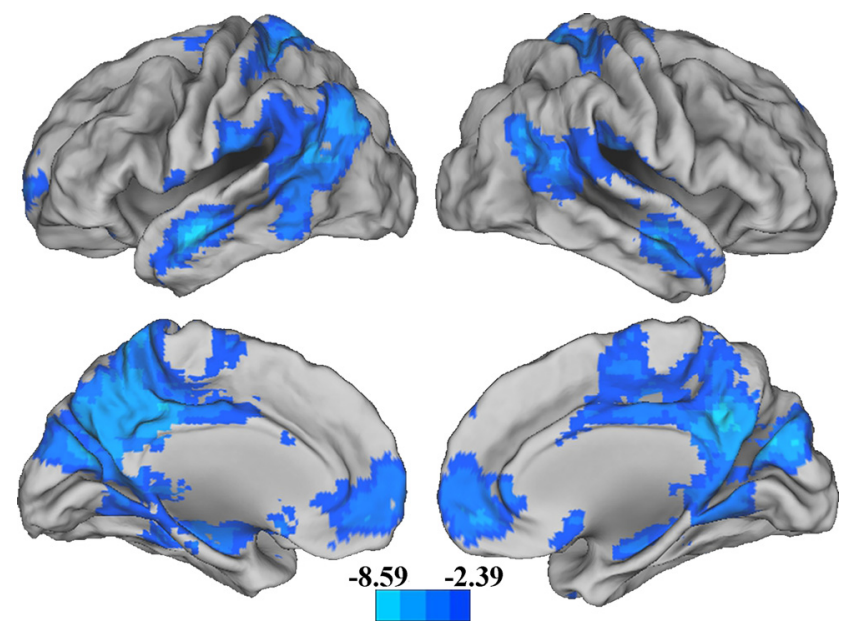

Figure 1. Cool colors represent regions that decrease activation as task difficulty increases (i.e., easy $>$ medium $>$ hard). This effect did not differ between APOE $\varepsilon 4$ noncarriers and carriers. Results were thresholded using a height threshold of $p<0.01$ and a nonparametric FWE cluster correction at $p<0.05$ using SnPM13. Scale indicates $t$-values.

as age increased. No other effects were significant ( $p$ values $>0.143$; Table 2).

\section{Modulation of activation to task difficulty}

Results of the whole-brain analysis on BOLD modulation in response to task difficulty (i.e., a test on the intercept) revealed a large cluster that included the bilateral cuneus and precuneus, bilateral lateral temporal gyrus, and bilateral medial prefrontal cortex. These regions exhibited significant down modulation in response to increasing difficulty (Fig. 1; Table 3, top). Cool colors represent regions that decrease activation as task difficulty increases (i.e., easy $>$ medium $>$ hard). Importantly, there was no significant effect of APOE genotype. Therefore, both groups down modulated a large cluster of default mode regions to a similar extent. No regions exhibited significant up modulation in response to increasing cognitive challenge. Results are unchanged after adjusting for either mean cortical thickness or mean cortical (intracranial adjusted) volume.

\section{Age effects on modulation in response to difficulty vary by APOE group}

Our primary hypothesis was that age-related differences in modulation in response to difficulty would differ by APOE group such that $\varepsilon 4+$ individuals would evidence exacerbated decline in modulation with age compared with the nonrisk $\varepsilon 4-$ individuals. In support of this hypothesis, the voxelwise whole-brain analysis revealed a robust interaction between age and APOE in a large cluster localized primarily to the bilateral precuneus (Table 3 bottom; Fig. 2A). Cool colors represent regions where the effect of age on BOLD modulation in response to difficulty differs by APOE genotype (i.e., age $\times$ APOE interaction). This interaction indicates that within this cluster, APOE $\varepsilon 4+$ individuals (Fig. $2 B$, filled triangles and solid line) reduce down modulation in response to task difficulty with age to a greater extent than $\varepsilon 4-$ individuals (Fig. 2B, open triangles and dashed line). To better understand the pattern of APOE-related differences in lifespan trajectories of down modulation, we used the significant interaction cluster as a mask to assess age-related differences in modulation in the $\varepsilon 4-$ group. Positive and negative contrasts of the simple effect of age on modulation in this cluster revealed no significant effects of age on modulation (Fig. 2C). Thus, $\varepsilon 4-$ individuals exhibit age-invariant modulation in response to difficulty in this cluster, whereas $\varepsilon 4+$ individuals exhibit a significantly steeper decline in modulation across the lifespan (Fig. $2 D)$. Incrementing down modulation in response to difficulty is shown as lighter to darker blues in Figure 2, $C$ and $D$.

Finally, to test whether the age and APOE interaction in the bilateral precuneus region was localized within the regions that responded to task difficulty overall in these two groups, we masked the significant interaction with the regions that down modulated in response to task difficulty (Fig. 1). The results revealed that the interaction was almost completely isolated to regions in which both $\varepsilon 4-$ and $\varepsilon 4+$ individuals down modulate. In sum, both $\varepsilon 4-$ and $\varepsilon 4+$ individuals successfully down modulate in response to increasing cognitive challenge; however, whereas the $\varepsilon 4-$ group exhibited no age-related decline in modulation, the APOE $\varepsilon 4+$ risk group showed a significantly greater agerelated decrease in precuneus down modulation.

\section{Relationship between modulation in response to difficulty and behavior}

To investigate the association between modulation in response to difficulty and cognition and to examine whether group differences were present in these associations, we conducted hierarchical multiple linear regressions on mean task accuracy and on associative memory. In the first model, we included effects of age, APOE, and modulation, and in the second hierarchical model we added all two-way and three-way interactions.

\section{Task accuracy}

There was a significant negative main effect of modulation $(\beta=$ $\left.-0.266, t_{(58)}=-2.103, p=0.040\right)$ on task accuracy such that as down modulation increased, task performance increased (Fig. $3 A)$. There were no significant main effects of age or APOE $(p$ values $>0.478)$, no significant $\Delta R^{2}$ when adding the interaction terms $\left(\Delta R^{2}=0.076, F_{(4,54)}=1.216, p=0.315\right)$, and no significant interactions with modulation ( $p$ values $>0.504)$. These results indicate that greater down modulation, in all individuals regardless of age or genetic group, was beneficial to performance.

\section{Associative memory}

The analysis on the Memory for Names Delayed test revealed a significant negative main effect of age $\left(\beta=-0.531, t_{(58)}=\right.$ $-5.041, p<0.001)$ and a significant negative main effect of modulation $\left(\beta=-0.238, t_{(58)}=-2.257, p=0.028\right.$; Fig. $\left.3 B\right)$. There was again no significant effect of $\operatorname{APOE}\left(t_{(58)}=0.309, p=0.758\right)$, no significant $\Delta R^{2}$ when adding the interaction terms $\left(\Delta R^{2}=\right.$ $\left.0.044, F_{(4,54)}=1.006, p=0.413\right)$, and no significant interactions with modulation ( $p$ values $>0.128$ ). These results indicate that individuals who show greater down modulation in response to difficulty perform better on an out-of-scanner measure of nameface associative memory, demonstrating that greater modulation is a beneficial attribute.

Last, the analysis of the Verbal Paired Associates Cued Recall test revealed only a significant main effect of age $(\beta=-0.539$, $\left.t_{(58)}=-4.925, p<0.001\right)$, but no other significant main effects ( $p$ values $>0.235$; Fig. $3 C$ ), no significant $\Delta R^{2}$ when adding the interaction terms $\left(\Delta R^{2}=0.056, F_{(4,54)}=1.190, p=0.326\right)$, and no significant interactions with modulation ( $p$ values $>0.062$ ). The combination of results provides evidence that, regardless of age or APOE status, greater down modulation is related to better performance on an out-of-scanner associative memory task and the in-scanner distance judgment task. In Figure $3 A-C$, solid lines indicate a significant effect, and the dashed line represents a nonsignificant effect. 
Table 3. Cluster peaks for task modulation in response to difficulty (top) and interaction between age and APOE on task modulation in response to difficulty (bottom)

\begin{tabular}{|c|c|c|c|c|c|c|c|}
\hline & \multirow[b]{2}{*}{$\mathrm{BA}$} & \multirow[b]{2}{*}{$k$} & \multicolumn{3}{|c|}{$\begin{array}{l}\text { MNI } \\
\text { coordinates }\end{array}$} & \multirow[b]{2}{*}{$t$} & \multirow{2}{*}{$\begin{array}{l}\text { Cluster } \\
\text { FWE } \\
p \text { value }\end{array}$} \\
\hline & & & $X$ & $y$ & $Z$ & & \\
\hline \multicolumn{8}{|l|}{ Decreased activation (deactivation) [easy $>$ medium $>$ hard] } \\
\hline $\mathrm{R} / \mathrm{L}$ precuneus, $\mathrm{R} / \mathrm{L}$ cuneus, $\mathrm{R} / \mathrm{L}$ middle cingulate, $\mathrm{R} / \mathrm{L}$ posterior cingulate, & $21,7,23,18,31,10$ & 14,135 & -54 & -6 & -18 & 8.59 & 0.0002 \\
\hline \multirow[t]{5}{*}{$\mathrm{R} / \mathrm{L}$ medial frontal gyrus, $\mathrm{R} / \mathrm{L}$ middle temporal gyrus } & & & 12 & -51 & 33 & 8.28 & \\
\hline & & & -3 & -69 & 21 & 7.62 & \\
\hline & & & 9 & -81 & 24 & 7.52 & \\
\hline & & & -3 & -60 & 51 & 6.35 & \\
\hline & & & 9 & 51 & 3 & 5.08 & \\
\hline \multicolumn{8}{|l|}{ Interaction between age and $\mathrm{APOE}$} \\
\hline \multirow[t]{3}{*}{$\mathrm{R} / \mathrm{L}$ precuneus, $\mathrm{R} / \mathrm{L}$ postcentral gyrus, $\mathrm{L}$ superior parietal cortex, $\mathrm{L}$ middle cingulate gyrus } & 7,5 & 992 & -9 & -51 & 66 & 3.77 & 0.029 \\
\hline & & & -9 & -51 & 57 & 3.67 & \\
\hline & & & 39 & -24 & 39 & 3.39 & \\
\hline
\end{tabular}

Note: The Statistical nonParametric Mapping toolbox version 13 (SnPM) was used to calculate cluster thresholds for the effect of difficulty and the interaction between age and AP0E on task modulation in response to difficulty. $R$, Right, L left: BA, Brodmann area.

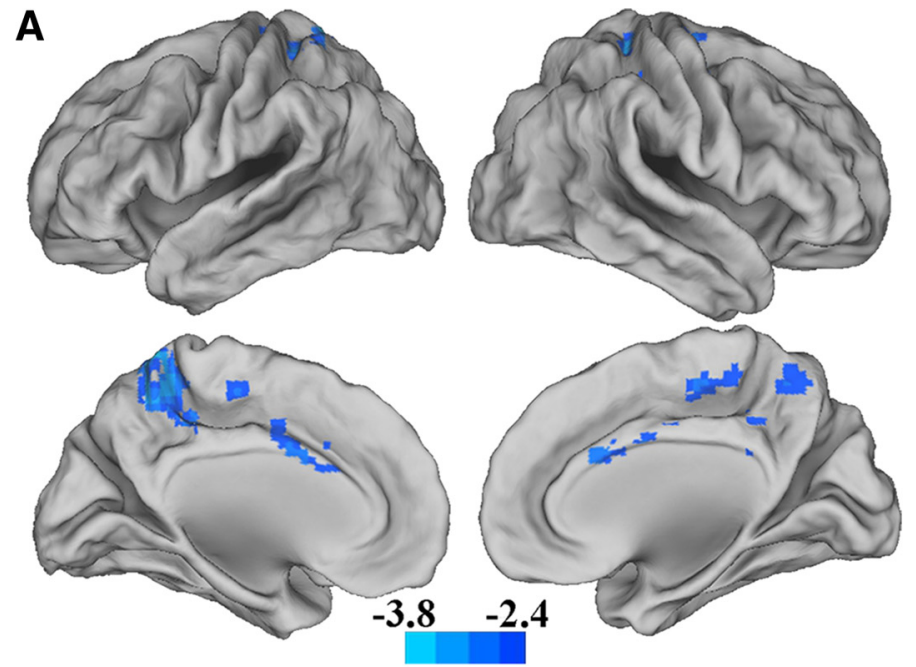

C

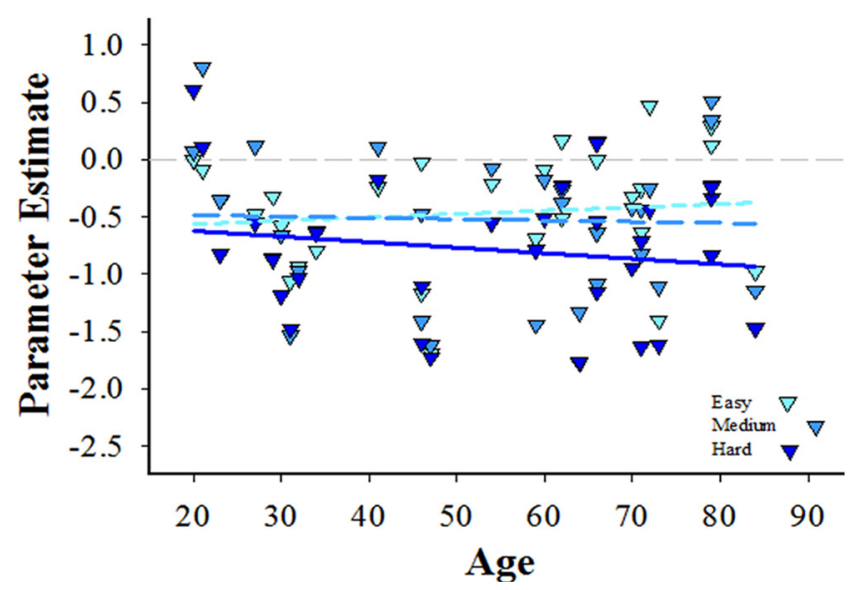

B

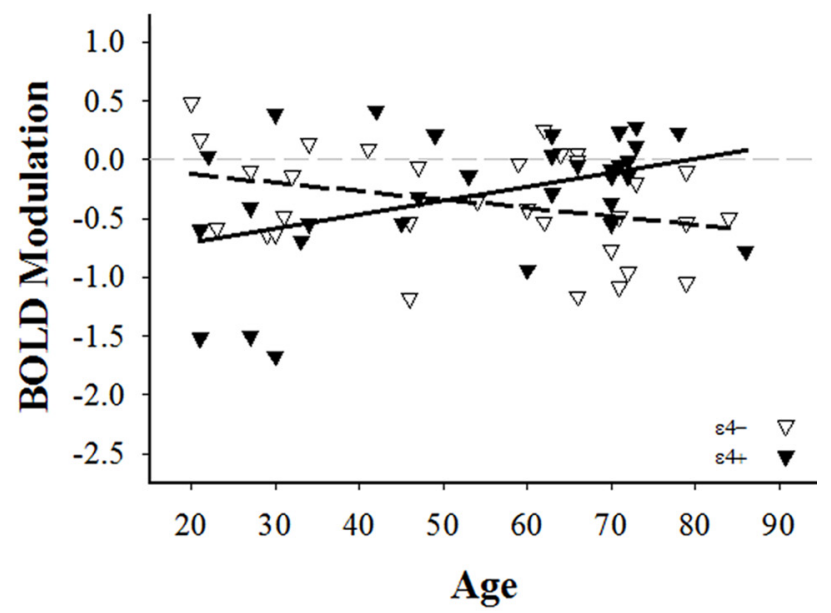

APOE \&4+

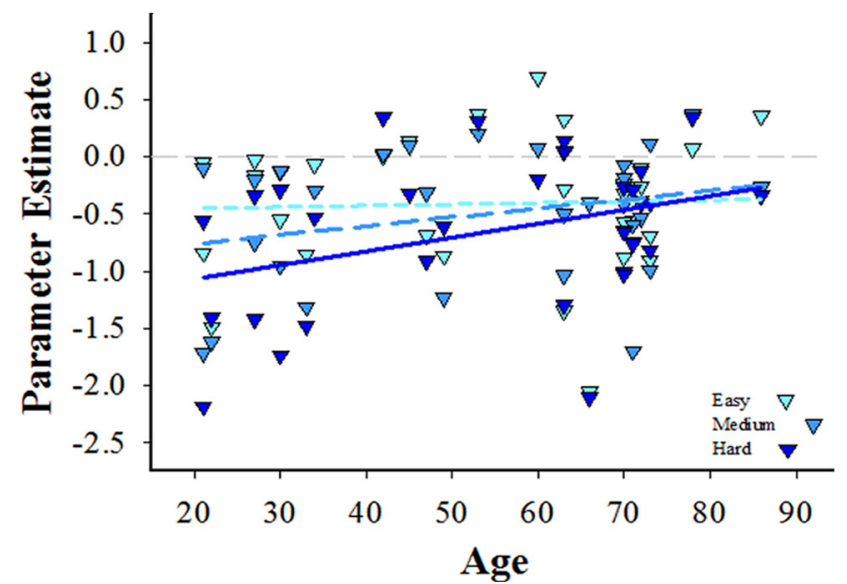

Figure 2. $\quad A$, Cool colors represent regions where the effect of age on BOLD modulation in response to difficulty differs by APOE genotype (i.e., age $\times$ APOE interaction) and includes bilateral precuneus and cingulate cortices. Scale indicates $t$-values. $B, A P O E \varepsilon 4-$ individuals exhibited no significant age-related differences in modulation across the lifespan (dashed line), whereas $\mathrm{APOE} \varepsilon 4+$ individuals decreased in the ability to modulate deactivation to difficulty with age relative to $\varepsilon 4-$ individuals (solid line). $C$, APOE $\varepsilon 4-$ individuals successfully down modulate in response to increasing task difficulty, and this effect is age invariant. D, APOE $\varepsilon 4+$ individuals also successfully down modulate in response to increasing task difficulty; however, this ability weakens across the lifespan compared with $\varepsilon 4+$ individuals.

\section{Discussion}

The present study tested the hypothesis that genetic risk for Alzheimer's disease modifies the effect of aging on brain function in response to cognitive challenge in a visuospatial processing task.
Results revealed that APOE $\varepsilon 4-$ and APOE $84+$ groups evidenced differential age effects on precuneus modulation in response to difficulty during a visuospatial distance judgment task. APOE $\varepsilon 4-$ individuals showed age-invariant modulation, 

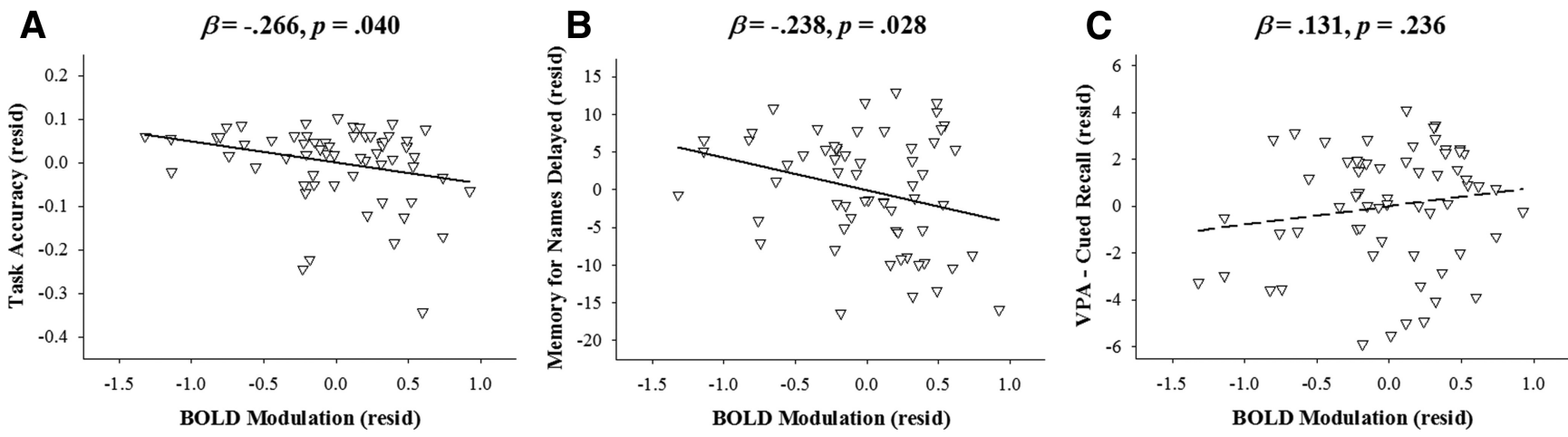

Figure 3. $A, B, G$ Geater BOLD modulation in response to difficulty is associated with better task accuracy $(\boldsymbol{A})$, and better face-name associative memory $(\boldsymbol{B})$, regardless of age or $A P O E$ genotype. $C$, However, this effect is not seen between BOLD modulation and verbal paired associate test performance. Note that all graphs plot residualized variables after removing the relationship with age and APOE.

whereas the demographically matched APOE $\varepsilon 4+$ group showed age-related decreases in modulation compared with the $\varepsilon 4-$ group. This effect was found in a sample that exhibited no behavioral differences on the in-scanner task, and, thus, it is less likely that these changes in modulation are driven by differential task difficulty between groups (Morcom et al., 2003; Snyder et al., 2011). Although more research is needed to specify the precise mechanisms underlying the effect of APOE 84 on precuneus BOLD modulation, APOE $\varepsilon 4$ alters lipid and $\beta$-amyloid transport and confers risk for hypometabolism, brain atrophy, and white matter hyperintensity accumulation (Reiman et al., 2005; Cherbuin et al., 2007; Liu et al., 2013), changes that may negatively impact neural efficiency and available resources.

While neural resources are thought to decline with normal aging, APOE $\varepsilon 4-$ individuals appear to maintain the ability to flexibly modulate the precuneus in response to increasing challenge in visuospatial processing across the lifespan. Thus, it is possible that age-related declines in modulation/resources represent the accumulation of significant risk factors for unhealthy aging, as opposed to a more general effect of aging. In contrast, APOE $\varepsilon 4+$ individuals fail to maintain precuneus modulation across the lifespan in response to cognitive challenge. Importantly, failure to modulate with age relates to, and potentially contributes to, poorer cognition, replicating previous research investigating modulation and age (Turner and Spreng, 2015; Rieck et al., 2017).

Age-related differences in down modulation between APOE $\varepsilon 4+$ and $\mathrm{APOE} \varepsilon 4$ - individuals were found in the bilateral precuneus. These results are interesting in the context of previous research involving the precuneus, a multifaceted heteromodal association region, which offers several possible insights into the nature of its involvement. This visuospatial distance judgment task requires the temporary encoding, storage, and retrieval of spatial relationships for successful performance. Thus, precuneus modulation in response to parametric levels of difficulty in the current study could simply reflect the role of the precuneus in visuospatial processing or it could represent an increased need to inhibit irrelevant information derived from long-term memory as task difficulty increases (for review, see Cavanna and Trimble, 2006). Similar to the current study, prior research has shown that healthy adults increase precuneus deactivation in response to hard judgments (Park et al., 2010); however, the effect of risk factors for $\mathrm{AD}$ on modulation were not explored.

While our results provide evidence that APOE alters precuneus modulation in response to visuospatial challenge, it will be important for future research to establish whether the effect of APOE on precuneus modulation is driven solely by the visuospatial demands of the task or whether it is a universal process driven more generally by increased task demands. Prior research using an $n$-back task, a task that increases both the amount of visuospatial information held in working memory as well as task difficulty, also found precuneus modulation differences in response to increasing difficulty between APOE groups (Wishart et al., 2006; Shine et al., 2015); however, several studies also found APOErelated group differences in a broader set of frontoparietal regions (Wishart et al., 2006; Chen et al., 2013; Yan et al., 2015). Within the current task, the amount of visuospatial information held within working memory is similar regardless of task difficulty, providing some evidence that the effect of APOE on precuneus modulation is driven primarily by the increase in cognitive challenge and a need to enhance focus on the task, as opposed to the amount of visuospatial information being held in working memory.

Second, the precuneus is also a major brain region implicated early in $\mathrm{AD}$ pathology and is known to evidence decreased metabolism in AD (Alexander et al., 2002); thus, altered precuneus modulation in this region may in part be driven by metabolic differences between APOE $\varepsilon 4-$ and APOE $\varepsilon 4+$ individuals. Relatedly, gray matter atrophy in precuneus is sensitive to amyloid load (Chételat et al., 2010), and $\beta$-amyloid accumulation in precuneus in healthy adults is associated with reduced frontal activation for subsequently remembered items (Kennedy et al., 2012). Because APOE 44 carriers are less able to clear amyloid, one potential mechanism driving decline in the ability to modulate activity in response to difficulty could be gray matter atrophy, which is linked to $\beta$-amyloid accumulation (Oh et al., 2015). However, controlling for both gray matter thickness and volume in the current study did not alter the results. It is also possible that APOE-related differences in brain activation may precede downstream changes associated with the accumulation of $\beta$-amyloid. The current sample of adults did not exhibit any signs of clinically significant cognitive decline or dementia, and thus, reduced precuneus modulation likely reflects a failure to appropriately recruit neural resources to accomplish challenging cognitive tasks as opposed to frank loss of brain tissue. Future research using longitudinal fMRI paradigms in healthy adults at risk for $\mathrm{AD}$ are needed to test this question.

Third, as the precuneus is an important functional hub in the brain, disruption of activity in precuneus is likely to lead to a variety of changes in activation and connectivity throughout the 
brain (Raichle et al., 2001; Fox et al., 2005). In APOE 44 carriers, decreased precuneus activation has been linked to different patterns of connectivity and complexity compared with their $\varepsilon 4-$ peers (Yang et al., 2014). More broadly, APOE $\varepsilon 4$ carriers have also been shown to exhibit altered functional connectivity across default-mode regions of the frontal, temporal, and parietal lobes (Dennis and Thompson, 2014), suggesting that APOE 84 also induces functional connectivity changes outside of the precuneus.

Reduced precuneus modulation within a sample of adults who are already at risk for $\mathrm{AD}$ (e.g., genetic and health factors) may be an important additional indicator of future decline, although it is unlikely that precuneus modulation would serve as an independent biomarker. The APOE $\varepsilon 4$ individuals at increased risk for $\mathrm{AD}$ in the current sample performed within the same range as their lower-risk peers on both the in-scanner task and the out-of-scanner associative memory task. However, precuneus modulation in response to difficulty was a significant predictor of cognitive performance. Therefore, the combination of challenging associative memory tasks and known risk factors (Sperling et al., 2014), along with measures of brain function such as precuneus modulation in response to difficulty or modulation in response to difficulty more generally, may improve the sensitivity with which adults destined for $\mathrm{AD}$ can be identified. Relatedly, as can be seen in Figure 2B, despite having a strong genetic risk factor for $\mathrm{AD}$, some individuals are still able to modulate precuneus activity in response to visuospatial challenge into older age. The current sample is not large enough to examine moderators of precuneus modulation within the $\varepsilon 4+$ group; however, future research aimed at examining what factors may preserve precuneus modulation despite genetic risk will be critical for delineating the precise role of $\mathrm{APOE} \varepsilon 4$ in precuneus modulation.

The results of the current study should be interpreted in light of several limitations. While our sample size is consistent with much prior literature (for review, see Trachtenberg et al., 2012), replicating the finding with a larger sample will be an important next step. It will be additionally important to verify these findings in a longitudinal sample. Prior research investigating the longitudinal change in activation found a similar pattern of results, namely that APOE $\varepsilon 4+$ individuals decline in activation across 5 years, whereas $\varepsilon 4-$ individuals exhibit the opposite response (Rao et al., 2015). However, this sample only included older adults and thus had a limited age range for comparison. Findings from the current study provide evidence that $\mathrm{APOE} \varepsilon 4$ is related to an altered lifespan trajectory in the ability of the brain to flexibly modulate activation in response to challenge. However, establishing within-person change across the lifespan will improve the current knowledge of how modulation changes with increasing age and how individual differences reduce or exacerbate the effect of APOE on brain function.

Understanding how risk factors for $\mathrm{AD}$ affect brain function and cognition in healthy adult samples is critical: to date, successful interventions aimed at ameliorating symptomatic $\mathrm{AD}$ remain to be established, necessitating the need to target future developments toward the nonsymptomatic, preclinical phase of the disease. The current results suggest that APOE $\varepsilon 4$ affects brain modulation in response to visuospatial processing across the lifespan. Thus, it may be necessary to target interventions to both older nonsymptomatic individuals and younger individuals who are decades from the earliest symptoms of the disease. The current findings also suggest that changes in the ability of the brain to dynamically modulate in response to cognitive challenge may serve as an additional risk for future age-related cognitive decline. However, further research is needed to elucidate the specificity and generalizability of these findings as well as to confirm the potential clinical significance.

\section{References}

Alexander GE, Chen K, Pietrini P, Rapoport SI, Reiman EM (2002) Longitudinal PET evaluation of cerebral metabolic decline in dementia: a potential outcome measure in Alzheimer's disease treatment studies. Am J Psychiatry 159:738-745. CrossRef Medline

Baciu M, Koenig O, Vernier MP, Bedoin N, Rubin C, Segebarth C (1999) Categorical and coordinate spatial relations: fMRI evidence for hemispheric specialization. Neuroreport 10:1373-1378. CrossRef Medline

Cattell R, Cattell A (1963) Test of "g": culture fair. Champaign, IL: Institute for Personality and Ability Testing.

Cavanna AE, Trimble MR (2006) The precuneus: a review of its functional anatomy and behavioural correlates. Brain 129:564-583. CrossRef Medline

Chen CJ, Chen CC, Wu D, Chi NF, Chen PC, Liao YP, Chiu HW, Hu CJ (2013) Effects of the apolipoprotein E $\varepsilon 4$ allele on functional MRI during n-back working memory tasks in healthy middle-aged adults. Am J Neuroradiol 34:1197-1202. CrossRef Medline

Cherbuin N, Leach LS, Christensen H, Anstey KJ (2007) Neuroimaging and APOE genotype: a systematic qualitative review. Dement Geriatr Cogn Disord 24:348-362. CrossRef Medline

Chételat G, Villemagne VL, Bourgeat P, Pike KE, Jones G, Ames D, Ellis KA, Szoeke C, Martins RN, O’Keefe GJ, Salvado O, Masters CL, Rowe CC (2010) Relationship between atrophy and beta-amyloid deposition in Alzheimer disease. Ann Neurol 67:317-324. CrossRef Medline

Corder EH, Saunders AM, Strittmatter WJ, Schmechel DE, Gaskell PC, Small GW, Roses AD, Haines JL, Pericak-Vance MA (1993) Gene dose of apolipoprotein E type 4 allele and the risk of Alzheimer's disease in late onset families. Science 261:921-923. CrossRef Medline

Craik F, Byrd M (1982) Aging and cognitive deficits: the role of attentional resources. In: Aging and cognitive processes (Craik FIM, Trehub S, eds), pp 199-211. New York: Plenum.

Dennis EL, Thompson PM (2014) Functional brain connectivity using fMRI in aging and Alzheimer's disease. Neuropsychol Rev 24:49-62. CrossRef Medline

Dennis NA, Browndyke JN, Stokes J, Need A, Burke JR, Welsh-Bohmer KA, Cabeza R (2010) Temporal lobe functional activity and connectivity in young adult APOE varepsilon4 carriers. Alzheimers Dement 6:303-311. CrossRef Medline

Educational Testing Service (1962) Vocabulary test II (V-2). Kit of factor referenced cognitive tests. Princeton, NJ: Educational Testing Services.

Eklund A, Nichols TE, Knutsson H (2016) Cluster failure: why fMRI inferences for spatial extent have inflated false-positive rates. Proc Natl Acad Sci U S A 113:7900-7905. CrossRef Medline

Filippini N, MacIntosh BJ, Hough MG, Goodwin GM, Frisoni GB, Smith SM, Matthews PM, Beckmann CF, Mackay CE (2009) Distinct patterns of brain activity in young carriers of the APOE-epsilon4 allele. Proc Natl Acad Sci U S A 106:7209-7214. CrossRef Medline

Filippini N, Ebmeier KP, MacIntosh BJ, Trachtenberg AJ, Frisoni GB, Wilcock GK, Beckmann CF, Smith SM, Matthews PM, Mackay CE (2011) Differential effects of the APOE genotype on brain function across the lifespan. Neuroimage 54:602-610. CrossRef Medline

Folstein MF, Folstein SE, McHugh PR (1975) “Mini-mental state.” A practical method for grading the cognitive state of patients for the clinician. J Psychiatr Res 12:189-198. CrossRef Medline

Fox MD, Snyder AZ, Vincent JL, Corbetta M, Van Essen DC, Raichle ME (2005) The human brain is intrinsically organized into dynamic, anticorrelated functional networks. Proc Natl Acad Sci U S A 102:9673-9678. CrossRef Medline

Frieden C, Garai K (2012) Structural differences between apoE3 and apoE4 may be useful in developing therapeutic agents for Alzheimer's disease. Proc Natl Acad Sci U S A 109:8913-8918. CrossRef Medline

Kennedy KM, Rodrigue KM, Devous MD Sr, Hebrank AC, Bischof GN, Park DC (2012) Effects of beta-amyloid accumulation on neural function during encoding across the adult lifespan. Neuroimage 62:1-8. CrossRef Medline

Kennedy KM, Rodrigue KM, Bischof GN, Hebrank AC, Reuter-Lorenz PA, Park DC (2015a) Age trajectories of functional activation under conditions of low and high processing demands: an adult lifespan fMRI study of the aging brain. Neuroimage 104:21-34. CrossRef Medline 
Kennedy KM, Reese ED, Horn MM, Sizemore AN, Unni AK, Meerbrey ME, Kalich AG Jr, Rodrigue KM (2015b) BDNF val66met polymorphism affects aging of multiple types of memory. Brain Res 1612:104-117. CrossRef Medline

Liu CC, Kanekiyo T, Xu H, Bu G (2013) Apolipoprotein E and Alzheimer disease: risk, mechanisms, and therapy. Nat Rev Neurol 9:106-118. CrossRef Medline

Matura S, Prvulovic D, Jurcoane A, Hartmann D, Miller J, Scheibe M, O’Dwyer L, Oertel-Knöchel V, Knöchel C, Reinke B, Karakaya T, Fußer F, Pantel J (2014) Differential effects of the ApoE4 genotype on brain structure and function. Neuroimage 89:81-91. CrossRef Medline

Mazaika PK, Hoeft F, Glover GH, Reiss AL (2009) Methods and software for fMRI analysis for clinical subjects. Neuroimage 47 [Suppl 1]:S58. CrossRef

Mondadori CR, de Quervain DJ, Buchmann A, Mustovic H, Wollmer MA, Schmidt CF, Boesiger P, Hock C, Nitsch RM, Papassotiropoulos A, Henke K (2007) Better memory and neural efficiency in young apolipoprotein E $\varepsilon 4$ carriers. Cereb Cortex 17:1934-1947. CrossRef Medline

Morcom AM, Good CD, Frackowiak RS, Rugg MD (2003) Age effects on the neural correlates of successful memory encoding. Brain 126:213-229. CrossRef Medline

Naveh-Benjamin M (2000) Adult age differences in memory performance: tests of an associative deficit hypothesis. J Exp Psychol Learn Mem Cogn 26:1170-1187. CrossRef Medline

Naveh-Benjamin M, Hussain Z, Guez J, Bar-On M (2003) Adult age differences in episodic memory: further support for an associative-deficit hypothesis. J Exp Psychol Learn Mem Cogn 29:826-837. CrossRef Medline

Naveh-Benjamin M, Guez J, Kilb A, Reedy S (2004) The associative memory deficit of older adults: further support using face-name associations. Psychol Aging 19:541-546. CrossRef Medline

Oh H, Steffener J, Razlighi QR, Habeck C, Liu D, Gazes Y, Janicki S, Stern Y (2015) $\mathrm{A} \beta$-related hyperactivation in fronto-parietal control regions in cognitively normal elderly. Neurobiol Aging 36:3247-3254. CrossRef Medline

Park DC, Polk TA, Hebrank AC, Jenkins LJ (2010) Age differences in default mode activity on easy and difficult spatial judgment tasks. Front Hum Neurosci 3:75. CrossRef Medline

Radloff LS (1977) A self-report depression scale for research in the general population. Appl Psychol Meas 1:385-401. CrossRef

Raichle ME, MacLeod AM, Snyder AZ, Powers WJ, Gusnard DA, Shulman GL (2001) A default mode of brain function. Proc Natl Acad Sci U S A 98:676-682. CrossRef Medline

Rao SM, Bonner-Jackson A, Nielson KA, Seidenberg M, Smith JC, Woodard JL, Durgerian S (2015) Genetic risk for Alzheimer's disease alters the five-year trajectory of semantic memory activation in cognitively intact elders. Neuroimage 111:136-146. CrossRef Medline

Reiman EM, Chen K, Alexander GE, Caselli RJ, Bandy D, Osborne D, Saunders AM, Hardy J (2005) Correlations between apolipoprotein E epsilon4 gene dose and brain-imaging measurements of regional hypometabolism. Proc Natl Acad Sci U S A 102:8299-8302. CrossRef Medline

Reuter-Lorenz PA, Cappell KA (2008) Neurocognitive aging and the compensation hypothesis. Curr Dir Psychol Sci 17:177-182. CrossRef
Rieck JR, Rodrigue KM, Boylan MA, Kennedy KM (2017) Age-related reduction of BOLD modulation to cognitive difficulty predicts poorer task accuracy and poorer fluid reasoning ability. Neuroimage 147:262-271. CrossRef Medline

Scheltens P, Blennow K, Breteler MM, de Strooper B, Frisoni GB, Salloway S, Van der Flier WM aria (2016) Alzheimer's disease. Lancet 388:505-517. CrossRef Medline

Schneider-Garces NJ, Gordon BA, Brumback-Peltz CR, Shin E, Lee Y, Sutton BP, Maclin EL, Gratton G, Fabiani M (2010) Span, CRUNCH, and beyond: working memory capacity and the aging brain. J Cogn Neurosci 22:655-669. CrossRef Medline

Shine JP, Hodgetts CJ, Postans M, Lawrence AD, Graham KS (2015) APOE- $\varepsilon 4$ selectively modulates posteromedial cortex activity during spatial perception and memory in young healthy adults. Sci Rep 5:16322. CrossRef Medline

Snyder AN, Bockbrader MA, Hoffa AM, Dzemidzic MA, Talavage TM, Wong D, Lowe MJ, O'Donnell BF, Shekhar A (2011) Psychometrically matched tasks evaluating differential fMRI activation during form and motion processing. Neuropsychology 25:622-633. CrossRef Medline

Sperling R, Mormino E, Johnson K (2014) The evolution of preclinical Alzheimer's disease: implications for prevention trials. Neuron 84:608-622. CrossRef Medline

Suri S, Mackay CE, Kelly ME, Germuska M, Tunbridge EM, Frisoni GB, Matthews PM, Ebmeier KP, Bulte DP, Filippini N (2015) Reduced cerebrovascular reactivity in young adults carrying the APOE $\varepsilon 4$ allele. Alzheimers Dement 11:648-657.e1. CrossRef Medline

Trachtenberg AJ, Filippini N, Mackay CE (2012) The effects of APOE- $\varepsilon 4$ on the BOLD response. Neurobiol Aging 33:323-334. CrossRef Medline

Turner GR, Spreng RN (2015) Prefrontal engagement and reduced default network suppression co-occur and are dynamically coupled in older adults: the default-executive coupling hypothesis of aging. J Cogn Neurosci 27:2462-2476. CrossRef Medline

Verghese PB, Castellano JM, Holtzman DM (2011) Apolipoprotein E in Alzheimer's disease and other neurological disorders. Lancet Neurol 10: 241-252. CrossRef Medline

Wechsler D (2009) Wechsler memory scale, Ed 4. San Antonio, TX: Pearson.

Wishart HA, Saykin AJ, McAllister TW, Rabin LA, McDonald BC, Flashman LA, Roth RM, Mamourian AC, Tsongalis GJ, Rhodes CH (2006) Regional brain atrophy in cognitively intact adults with a single APOE epsilon4 allele. Neurology 67:1221-1224. CrossRef Medline

Woodcock RW, McGrew KS, Mather N (2007) Woodcock Johnson III. Rolling Meadows, IL: Riverside.

Yan FX, Wu CW, Chao YP, Chen CJ, Tseng YC (2015) APOE- 84 allele altered the rest-stimulus interactions in healthy middle-aged adults. PLoS One 10:e0128442. CrossRef Medline

Yang AC, Huang CC, Liu ME, Liou YJ, Hong CJ, Lo MT, Huang NE, Peng CK, Lin CP, Tsai SJ (2014) The APOE varepsilon4 allele affects complexity and functional connectivity of resting brain activity in healthy adults. Hum Brain Mapp 35:3238-3248. CrossRef Medline 